\title{
CREENCIAS, ACTITUDES Y ANSIEDAD ANTE LA MUERTE EN UN EQUIPO MULTIDISCIPLINARIO DE CUIDADOS PALIATIVOS ONCOLÓGICOS
}

\author{
BELIEFS, ATTITUDES, DEATH ANXIETY IN A MULTIDISCIPLINARY TEAM ONCOLOGY \\ PALLIATIVE CARE
}

Leticia Ascencio Huertas, Silvia R. Allende Pérez y Emma Verastegui Avilés

Servicio de Cuidados Paliativos del Instituto Nacional de Cancerología, México D.F., México.

Resumen

En el ámbito de la salud, se ha puesto en evidencia la necesidad de profundizar en las actitudes y creencias ante la muerte en los propios profesionales ya que pueden influir en la calidad de los cuidados, especialmente las derivadas de procesos de muerte y duelo, por lo que el objetivo de la investigación fue explorar y analizar actitudes y creencias ante la muerte en el personal de salud en Cuidados Paliativos Oncológicos. Fue un estudio, transversal y descriptivo; se utilizó un muestreo intencional, los instrumentos utilizados fueron: Formato de datos personales y profesionales, Escala de Ansiedad ante la muerte de Templer, Creencias con relación al paciente terminal y Cuestionario de Actitudes ante la Muerte (CAM). Se analizaron los datos estadísticamente: análisis de frecuencias y correlación de Spearman, con una alta tendencia en considerar que es mejor que el paciente terminal fallezca en casa, en el uso de la morfina como tratamiento para el dolor en cáncer avanzado y recibir una atención integral. Dentro de las principales actitudes del personal de salud hacia el paciente terminal y la muerte se encontraron: mayor responsabilidad y atención hacia la vida, y promoción del crecimiento personal para aceptar su propia muerte.

Palabras clave: Creencias, actitudes, ansiedad ante la muerte, cuidados paliativos.

\section{Abstract}

In the field of health, has highlighted the need to delve into the attitudes and beliefs about death in the professionals because they can influence the quality of care, especially those derived from processes of death and mourning, for what the objective of the research was to explore and analyze attitudes and beliefs about death in health personnel Oncology Palliative Care. The study was a cross-sectional and descriptive, purposive sampling was used, the instruments used were format personal and professional, Anxiety Scale Templer's death, beliefs regarding the terminal patient and Attitudes Questionnaire Death (CAM). Data were analyzed statistically: Frequency analysis and Spearman correlation, with a high tendency to consider that is better than the terminal patient dies at home, in the use of morphine as a treatment for pain in advanced cancer and receiving comprehensive care. Among the main attitudes in the health staff towards the patient and death were found: more responsibility and attention to the promotion of life and personal growth to take his own life.

Key words: Beliefs, attitudes, death anxiety, palliative care.

\section{Correspondencia:}




\section{INTRODUCCIÓN}

El manejo de la muerte es muy complejo dentro del sistema de salud, principalmente en aquellos servicios de atención a pacientes con enfermedades crónico-degenerativas y en donde la muerte del paciente forma parte del proceso de atención cotidiano. La atención de este tipo de pacientes hace necesario considerar a la muerte, no únicamente como proceso biológico sino como un proceso multidimensional en el que la atención de los aspectos psicosociales, económicos y culturales son importantes de considerar. Estas necesidades se han visto reflejadas en la conformación de los servicios de cuidados paliativos cuya función principal es la atención global para el paciente con enfermedad terminal y su familia y en los que es necesario contar servicios interdisciplinarios La atención del binomio paciente-familia, es claramente el objeto de la atención del equipo sin embargo, los aspectos de incertidumbre, lo impredecible, lo incontrolable y el sufrimiento que acompañan a la muerte, alcanzan de alguna manera al personal de salud que están expuestos a interaccionar diariamente con dichos factores ${ }^{(1)}$. Los profesionales de la salud inevitablemente enfrentan sus propias limitaciones personales y profesionales y a plantearse la propia concepción de la muerte.

Tanto el pensamiento como la consciencia de muerte, son características exclusivamente humanas que provocan emociones intensas y una gama de actitudes y creencias que se manifiestan a través de distintas reacciones como desesperación, miedo y ansiedad, incluso ante la propia muerte ${ }^{(2)}$.

La ansiedad ante la muerte ha sido definida como una reacción emocional producida por la percepción de las señales de peligro o amenazas reales o imaginarias, a la propia existencia que puede desencadenarse por estímulos ambientales, situacionales o disposicionales, relacionados con la propia muerte o ajena ${ }^{(3)}$. Por otro, Heidegger expo- ne que la angustia ante la muerte es vivida como el miedo a la aniquilación y a la experiencia de la nada, lo cual causa temor ${ }^{(4)}$.

Las actitudes frente a la idea de la muerte han variado a través del tiempo y en las distintas sociedades y culturas. Lo que le confiere características únicas que están dadas por la historia personal de las perdidas vividas y a la forma de elaborar duelos frente a las mismas. También influyen la esperanza de vida y las creencias sobre lo que es un ser humano(5).

La actitud que cada cual adopte ante la muerte también dependerá de las creencias religiosas o agnósticas, de la concepción filosófica, así como de la capacidad para enfrentarnos con la realidad de la vida o con la realidad o no de la muerte. Dichas actitudes inciden directamente en cómo se plantea el acompañamiento de otros que mueren y esto tiene que ver con cómo experimentamos la situación de la muerte ajena. Para todo ser humano es importante prepararse para la situación de la muerte de otros.

En el ámbito de la salud es evidente la necesidad de abordar las emociones de los profesionales que pudieran influir en la calidad de los cuidados, especialmente aquellas experiencias derivadas de procesos de muerte y de duelo de pacientes y familiares. Hay autores que sugieren ${ }^{(6,7,8)}$ que es inevitable que aspectos emocionales del profesional de la salud incidan en la manera de comunicarse con el paciente, en la toma de decisiones, la capacidad de afrontamiento, resolución de los problemas y también en su integración en el conjunto asistencial ${ }^{(9,10)}$.

Los profesionales que laboran en las unidades de cuidados paliativos requieren de una alta competencia emocional ya que debe utilizar estrategias que den como resultado la satisfacción de las demandas de enfermos terminales y sus familiares al final de la vida ${ }^{(11)}$ En este aspecto es importante considerar para el cuidado 
del equipo de salud, que en la medicina actual el proceso de muerte se enmarca en el modelo occidental (donde la muerte se considera un enemigo), lo que genera reacciones emocionales de frustración, miedo, ansiedad y depresión ${ }^{(12)}$ que pudieran incidir en la calidad de la atención.

De acuerdo a Mok, Lee y Wong ${ }^{(13)}$ cuando el personal de salud asume la muerte como un proceso natural, los niveles de ansiedad generados por la muerte de los enfermos disminuye, debido a la comprensión del suceso terminal, su significado y al asumir las emociones propias que genera la muerte. Gala, Lupiani, Raja, Guillén, González, Villaverde y Sánchez ${ }^{(14)}$ y Pichardo y Diner ${ }^{(15)}$ reportan que corresponde al personal de salud acompañar al paciente y a su familia en el proceso de muerte, por lo es necesario que exista un adecuado manejo emocional y un interés genuino. Dentro de las principales consideraciones en el manejo de la ansiedad ante la muerte está el mantener un adecuado control emocional, comprensión de la muerte, de su significado y sostener una actitud serena y madura frente al paciente, familia y contexto hospitalario ${ }^{(16)}$.

Existen reportes como el de Collel, Limonero y Otero ${ }^{(17)}$ y Gómez Sancho ${ }^{(18)}$ que sugieren que la ansiedad que en el médico se genera ante la muerte de un paciente está dada por la confrontación con su propia muerte; que cambian sus actitudes y conductas ante la conclusión de un estadio terminal ${ }^{(19)}$ y que son los médicos los que ha presentado más miedos ante la muerte que los propios pacientes y que se ha vista reflejado en la disminución de la motivación desafiante de sus habilidades e inexplicables cambios de humor ${ }^{(20)}$.

Diversas investigaciones han intentado determinar cuáles son las variables que influyen en la ansiedad ante la muerte: género, edad $d^{(11,17,21,22)}$, estado civil ${ }^{(2)}$ psicoemocionales ${ }^{(7,}{ }^{23-25)}$ y déficit de formación profesional ${ }^{(18)}$.
Por lo que con el propósito de conocer más acerca de los factores que pueden influir en las actitudes, creencias y ansiedad del personal de salud ante la muerte, en un equipo multidisciplinario de cuidados paliativos que maneja pacientes con cáncer avanzado en el Instituto Nacional de Cancerología.

\section{MÉTODO}

Se utilizó un diseño no experimental, transversal y correlacional a través de un muestreo no probilistico y opinático, cuya población estuvo compuesta por el equipo multidisciplinario que prestó sus servicios profesionales en el Servicio de Cuidados Paliativos del Instituto Nacional de Cancerología de Marzo del 2011 a Febrero 2012, cuyos criterios de eliminación fueron no concluir la aplicación completa de todos los instrumentos psicológicos y/o que decidieran no participar en el estudio.

\section{Participantes}

Treinta y un profesionales de la salud de diversas especialidades, que prestaron sus servicios profesionales durante 12 meses (de marzo del 2011 a febrero del 2012) en el Servicio de Cuidados Paliativos del Instituto Nacional de Cancerología.

\section{Variables}

Las variables estuvieron categorizadas de la siguiente manera:

1. Variables generales: Edad, Género, Estado Civil.

2. Variables profesionales: Disciplina Profesional en Salud, Grado Académico, Años de experiencia Clínica, Tiempo (años) dedicado a la atención del paciente con cáncer, Tiempo (meses) dedicado de atención al paciente terminal. 
3. Creencias. Firme acertamiento y conformidad en algo o un estado disposicional adquirido, que causa un conjunto coherente de respuestas y que está determinado por un objeto o situación objetiva aprehen$\operatorname{didos}^{(26)}$ en el presente estudio se consideró esta definición con respecto al paciente terminal y su familia, los tratamientos, y su manejo.

4. Actitudes. Respuestas evaluativas relativamente estables, en relación a un objeto, que tiene consecuencias cognitivas afectivas y comportamentales $^{(27)}$ con respecto a las actitudes ante la muerte se consideran principalmente las dificultades para nombrar a la misma muerte, no mirar frente a frente al paciente, el aumento de la atención tecnológica, el encarnizamiento terapéutico y una formación adecuada en la atención del paciente terminal ${ }^{(14)}$.

5. Ansiedad ante la muerte. Reacción emocional producida por la percepción de las señales de peligro o amenazas reales o imaginarias, a la propia existencia que puede desencadenarse por estímulos ambientales, situacionales o disposicionales, relacionados con la propia muerte o ajena ${ }^{(3)}$ Y por estímulos internos de la persona, como pensamientos o evocación de imágenes relacionados con la muerte propia o ajena ${ }^{(27)}$.

\section{Instrumentos}

Fueron 3 los instrumentos de evaluación utilizados en la investigación:

\section{Cuestionario de Actitudes ante la Muerte (CAM)}

El Cuestionario de Actitudes ante la Muerte (CAM), está compuesto por 33 reactivos, con 2 alternativas de respuesta (acuerdo o desacuerdo), donde un total de 141 puntos o más corresponde a actitud positiva hacia la muerte y puntajes inferiores a actitud negativa. Para su interpretación está dividido en 6 dimensiones que corresponden a diferentes actitudes ante la muerte: de evitación, de aceptación, de temor, basadas en las creencias de que la muerte es un pasaje o tránsito, fundamentadas en la concepción de la muerte como una salida o solución y otra que involucra la perspectiva profesional. Con un alpha de Cronbach de $0,7139^{(29)}$.

\section{Cuestionario de Creencias con relación al paciente terminal}

Es un instrumento construido con quince ítems con creencias sociales $y$ emociones relacionadas con la muerte $y$ el paciente terminal. Se puntúa del 1 al 7 escala donde el 1 corresponde a totalmente en desacuerdo y 7 a totalmente de acuerdo $^{(30)}$. Es un instrumento meramente exploratorio creado por Collel, quien es experto en el estudio de creencias y actitudes ante la muerte y debido a que no existen instrumentos que evalúen las creencias por la dificultad del constructo se consideró dicha instrumento como recurso metodológico para explorar las creencias en el personal de salud de Cuidados Paliativos.

\section{Escala de Ansiedad ante la muerte de Templer}

La escala consiste en un cuestionario auto-administrable, que fue traducido $y$ adaptado al español por Ramos en 1982 y adaptada población mexicana por Tomás-Sábado y Gómez- Benito ${ }^{(28)}$ y RiveraLedesma y Montero-López ${ }^{(31)}$ La escala de respuesta se es tipo Likert de cuatro puntos: nunca o casi nunca, algunas veces, la mayor parte del tiempo, todo el tiempo, en donde el puntaje mínimo total 
es de 15 (mínima ansiedad) y el puntaje máximo total es 60 (máxima ansiedad) y está compuesta por 3 sub escalas: miedo a la muerte, miedo a la agonía o enfermedad y miedo a que la vida llegue a su fin. La consistencia interna del instrumento, medida con el alfa de Cronbach, en una muestra indica para adultos mayores $(0,86)$ y para estudiantes $(0,83)$. Se obtuvieron propiedades psicométricas que la hacen recomendable para su uso en adultos mayores mexicanos. La consistencia interna del instrumento con el alfa de Cronbach fue de $0,86^{(31)}$.

\section{Procedimiento}

El proceso de aplicación se llevó a cabo en el servicio de Cuidados Paliativos del Instituto Nacional de Cancerología en una sesión conjunta en donde se encontraba todo el personal del servicio, posteriormente se procedió a informarles el objetivo del estudio y se les entrego un consentimiento informado. El investigador principal entrego personalmente los instrumentos en un sobre cerrado a cada uno de los miembros del personal y se solicitó la devolución de los mismos nuevamente en un sobre cerrado directamente al investigador principal, con la finalidad de garantizar el anonimato.

Una vez realizada la aplicación de los instrumentos, se procedió la calificación de los mismos, así como la codificación de los datos; que posteriormente fueron sometidos a análisis estadísticos: Análisis de frecuencias y Correlación de Spearman.

\section{RESULTADOS}

La muestra estuvo compuesta por 35 sujetos de los cuales fueron eliminados 4 debido a que no contestaron completos los instrumentos o bien decidieron no participar voluntariamente en el estudio, por lo que la muestra total fue de 31 su- jetos, cuyas edades se encuentran de 26 a 30 años (41,9\%); con respecto al sexo se encontró que el 90,3\% pertenece al sexo femenino y $77,4 \%$ solteros. En las variables profesionales se encontró que la muestra estuvo compuesta por diversas profesiones del área de la salud: medicina, psicología, nutrición, enfermería y trabajo social en su mayoría con el grado de licenciatura. De acuerdo a los años de experiencia se encontró que los participantes cuentan con experiencia clínica el $45,1 \%$ y $744,2 \%$ con paciente oncológico con menos de 5 años y 35,6\% con paciente terminal con menos de 6 meses (Ver tabla 1).

En relación al Cuestionario de creencias con respecto al paciente terminal se encontraron como datos significativos que el personal de salud de cuidados paliativos: están en desacuerdo con las creencias acerca de que a un enfermo moribundo de cáncer es una persona que muere con mucho dolor $(54,8 \%)$, que se le puede ayudar muy poco $(100 \%)$, que presenta muchos síntomas difíciles de tratar $(64,5 \%)$, que el área de enfermería puede intervenir muy poco en el proceso de morir $(100 \%)$, que es preferible ocultar al enfermo que está muriendo (100\%), que lo mejor es decirle a la familia que su ser querido está muriendo y esconder la verdad al enfermo (100\%) y que la morfina crea adicción y anula la voluntad del enfermo terminal (90,3\%). Se encuentran de acuerdo con las creencias con respecto a: que tanto para el familiar como para el enfermo, lo mejor es que muera en su casa $(90,4 \%)$, que la morfina se utiliza para el tratamiento del dolor $(87,1 \%)$, que es muy importante para el paciente terminal recibir apoyo psicológico y espiritual $(93,5 \%)$, que la boca seca, el estreñimiento, el cansancio y el desgano son síntomas habituales en el paciente terminal $(93,5 \%)$ y lo que más le preocupa al enfermo terminal en el proceso del morir es el control de síntomas (71\%). Dentro de 


\section{Tabla 1. Frecuencias y Porcentajes de las variables generales y profesionales}

\section{Edad}

$\begin{array}{ccc}\text { 20-25 años } & 5 & 16,2 \\ \mathbf{2 6 - 3 0} \text { años } & 13 & 41,9 \\ \mathbf{3 1 - 4 0} \text { años } & 7 & 22,6 \\ \mathbf{4 6 - 5 0} \text { años } & 2 & 6,4 \\ \mathbf{5 1 - 5 5} \text { años } & 2 & 6,4 \\ \mathbf{5 6} \text { a } \mathbf{6 0} \text { años } & 2 & 6,4\end{array}$

Sexo

$\begin{array}{ccc}\text { Femenino } & 28 & 90,3 \\ \text { Masculino } & 3 & 9,7\end{array}$

\section{Estado Civil}

Solter@

Casad@

Divorciad@

Viud@

\section{4}

4

2

1
77,4

12,9

6,5

3,2

Área Profesional

Medicina

Psicología

Trabajo Social

Enfermería

Nutrición

Odontología

$\begin{array}{cc}9 & 29,0 \\ 11 & 35,5 \\ 2 & 6,5 \\ 4 & 12,9 \\ 4 & 12,9 \\ 1 & 3,2\end{array}$

Grado de Estudios

Licenciatura

Maestría

Especialidad

Post Doctorado

Sub Especialidad
18

5

3

1

4
58,1

16,1

9,7

3,2

12,9 
Frecuencia

Porcentaje

Años de Experiencia Clínica

$\begin{array}{ccc}\mathbf{1 - 5} \text { años } & 14 & 45,1 \\ \mathbf{6 - 1 0} \text { años } & 9 & 29 \\ \mathbf{1 1 - 1 5} \text { años } & 2 & 6,5 \\ \mathbf{1 6} \text { a } \mathbf{2 0} \text { años } & 1 & 3,2 \\ \mathbf{2 1} \text { a } \mathbf{2 5} \text { años } & 1 & 3,2 \\ \mathbf{2 6} \text { a más años } & 4 & 12,9\end{array}$

Años de Experiencia en la atención de paciente con cáncer

\begin{tabular}{ccc} 
0-5 años & 23 & 74,2 \\
6-10 años & 1 & 3,2 \\
11-15 años & 2 & 6,5 \\
$\mathbf{1 6}$ a 20 años & 1 & 3,2 \\
$\mathbf{2 1}$ a 25 años & 1 & 3,2 \\
26 a 30 años & 3 & 9,7 \\
\hline
\end{tabular}

Tiempo de atención al paciente terminal

\begin{tabular}{ccc} 
0 a 6 meses & 11 & 35,6 \\
7 a12 meses & 7 & 22,6 \\
$\mathbf{1 3}$ a18 meses & 5 & 16,1 \\
$\mathbf{1 9}$ a 24 meses & 1 & 3,2 \\
$\mathbf{2 5}$ a 36 meses & 3 & 9,7 \\
$\mathbf{6 1}$ a 72 meses & 1 & 3,2 \\
$\mathbf{9 7}$ a 120 meses & 1 & 3,2 \\
$\mathbf{2 4 1}$ a 300 meses & 1 & 3,2 \\
$\mathbf{3 0 1}$ a 360 meses & 1 & 3,2 \\
\hline
\end{tabular}

las creencias que se encuentran neutrales en el equipo de salud de Cuidados Paliativos es con respecto a que el dolor es el síntoma más difícil de tratar en el paciente terminal, que el familiar no quiere que el enfermo se enteré de que está muriendo y que lo que más preocupa al familiar son los aspectos emocionales, como se muestra en la tabla 2.
En la tabla 3 se presentan los porcentajes las sub escalas del Cuestionario de Actitudes ante la Muerte (CAM); encontrándose puntajes altos de desacuerdo en las sub escalas de Evitación (valora a la muerte como pasaje o tránsito hacia una vida mejor); Temor (Temor a la muerte, a contraer la enfermedad y a enfrentarse con la familia de un moribundo); Pasaje 
Tabla 2. Frecuencias y Porcentajes del Cuestionario de Creencias con relación al Paciente Terminal

\begin{tabular}{|c|c|c|c|c|c|c|c|c|}
\hline & \multirow[b]{2}{*}{ Reactivos } & \multicolumn{3}{|c|}{ Desacuerdo } & \multicolumn{2}{|c|}{ Neutral } & \multicolumn{2}{|c|}{ De Acuerdo } \\
\hline & & 1 & 2 & 3 & 4 & 5 & 6 & 7 \\
\hline & & $\mathrm{N} \%$ & $\mathrm{~N} \%$ & $\mathrm{~N} \%$ & N \% & $\mathrm{N} \%$ & $\mathrm{~N} \%$ & $\mathrm{~N} \%$ \\
\hline 1 & $\begin{array}{l}\text { Un enfermo moribundo de cáncer es una } \\
\text { persona que muere con mucho dolor físico }\end{array}$ & $9 / 29$ & $5 / 16,1$ & $3 / 9,7$ & $6 / 19,4$ & $3 / 9,7$ & $2 / 6,5$ & $3 / 9,7$ \\
\hline 2 & $\begin{array}{l}\text { A un enfermo terminal se le puede ayudar } \\
\text { muy poco }\end{array}$ & $27 / 87,1$ & $3 / 9,7$ & $1 / 3,2$ & & & & \\
\hline 3 & $\begin{array}{l}\text { El enfermo terminal es un enfermo que presen- } \\
\text { ta muchos síntomas difícil de tratar }\end{array}$ & $4 / 12,9$ & $9 / 29$ & $7 / 22,6$ & $3 / 9,7$ & $5 / 16,1$ & $1 / 3,2$ & $2 / 6,5$ \\
\hline 4 & $\begin{array}{l}\text { Enfermería puede intervenir muy poco en el } \\
\text { proceso de morir de un enfermo terminal }\end{array}$ & $25 / 80,6$ & $5 / 16,1$ & $1 / 3,2$ & & & & \\
\hline 5 & $\begin{array}{l}\text { Es preferible ocultar al enfermo que está } \\
\text { muriendo }\end{array}$ & $29 / 93,5$ & $1 / 3,2$ & $1 / 3,2$ & & & & \\
\hline 6 & $\begin{array}{l}\text { Lo mejor es decirle al familiar que su ser } \\
\text { querido se está muriendo y esconder la verdad } \\
\text { al enfermo }\end{array}$ & $29 / 93,5$ & $2 / 6,5$ & & & & & \\
\hline 7 & $\begin{array}{l}\text { Tanto para el familiar como para el enfermo, lo } \\
\text { mejor es que muera en su casa }\end{array}$ & & $1 / 3,2$ & & $2 / 6,5$ & $3 / 9,7$ & $7 / 22,6$ & $18 / 58,1$ \\
\hline 8 & $\begin{array}{l}\text { La morfina se utiliza para el tratamiento del } \\
\text { dolor }\end{array}$ & $2 / 6,5$ & $1 / 3,2$ & $1 / 3,2$ & & $3 / 9,7$ & $5 / 16,1$ & $19 / 61,3$ \\
\hline 9 & $\begin{array}{l}\text { Es muy importante para el enfermo terminal, } \\
\text { recibir apoyo psicológico y espiritual }\end{array}$ & $1 / 3,2$ & & & $1 / 3,2$ & & $1 / 3,2$ & $28 / 90,3$ \\
\hline 10 & $\begin{array}{l}\text { El dolor es el síntoma más difícil de tratar en el } \\
\text { enfermo moribundo }\end{array}$ & $4 / 12,9$ & $4 / 12,9$ & $6 / 19,4$ & $3 / 9,7$ & $8 / 25,8$ & $5 / 16,1$ & $1 / 3,2$ \\
\hline 11 & $\begin{array}{l}\text { La boca seca, el estreñimiento, el cansancio } \\
\text { y el desgano son síntomas habituales en el } \\
\text { paciente terminal }\end{array}$ & & & & $2 / 6,5$ & $4 / 12,9$ & $8 / 25,8$ & $17 / 54,8$ \\
\hline 12 & $\begin{array}{l}\text { El familiar no quiere que el enfermo se enteré } \\
\text { de que está muriendo }\end{array}$ & $4 / 12,9$ & $1 / 3,2$ & $2 / 6,7$ & $8 / 25,8$ & $9 / 29$ & $4 / 12,9$ & $2 / 6,5$ \\
\hline 13 & $\begin{array}{l}\text { La morfina crea adicción y anula la voluntad } \\
\text { del enfermo terminal }\end{array}$ & $25 / 80,6$ & $2 / 6,5$ & $1 / 3,2$ & $3 / 9,7$ & & & \\
\hline 14 & $\begin{array}{l}\text { Lo que más le preocupa al familiar son los } \\
\text { aspectos emocionales }\end{array}$ & $3 / 9,7$ & $4 / 12,9$ & $3 / 9,7$ & $6 / 19,4$ & $8 / 25,8$ & $6 / 19,4$ & $1 / 3,2$ \\
\hline 15 & $\begin{array}{l}\text { Lo que más preocupa al enfermo terminal } \\
\text { en el proceso de morir es el control de los } \\
\text { síntomas }\end{array}$ & $3 / 9,7$ & $3 / 9,7$ & $1 / 3,2$ & $2 / 6,5$ & $4 / 12,9$ & $6 / 19,4$ & $12 / 38,7$ \\
\hline
\end{tabular}


Tabla 3. Porcentajes del Cuestionario de Actitudes Ante la Muerte (CAM)

Porcentajes

Desacuerdo Acuerdo

Evitación Pensar en la muerte es perder el tiempo

$\begin{array}{cc}93,5 & 6,5 \\ 87,1 & 12,9 \\ 87,1 & 12,9 \\ 90,3 & 9,7 \\ 96,8 & 3,2\end{array}$

Aceptación La aceptación de la muerte me ayuda a tener más responsabilidad ante la

vida

Considero morboso pensar deliberadamente en mi muerte inevitable

Yo realmente prefiero no pensar en la muerte

2,9

No he pensado hasta ahora en la muerte como una posibilidad rea

3,2

Las personas sólo deberían pensar en la muerte cuando son viejos

Mi vida tiene más significado porque yo acepto el hecho de mi muerte

He pensado en mi muerte como un hecho imposible

Reconocer mi muerte como un hecho inevitable me ayuda a mi crecimiento personal

Me siento más libre al aceptar mi muerte

$\begin{array}{cc}9,7 & 90,3 \\ 9,7 & 90,3 \\ 93,5 & 6,5 \\ & \\ 12,9 & 87,1 \\ 12,9 & 87,1\end{array}$

Temor

La posibilidad de mi propia muerte me despierta ansiedad

77,4

22,6

Me siento perturbado cuando pienso lo corta que es la vida

Yo temo morir joven

Encuentro difícil encarar la muerte

Pienso con temor en la posibilidad de contraer una enfermedad que me lleve

irremediablemente a la muerte

Pasaje

Yo pienso que después de la vida habrá un lugar mejor

41,9

58,1

Pienso que viviré después de la muerte

Veo la muerte como un paso a la eternidad

51,6

48,4

Yo espero con placer la vida después de la muerte

67,7

32,3

Después de la muerte encontraré la felicidad

80,6

19,4

Salida

La muerte puede ser una salida a la carga de la vida

Perspectivas He vivido la muerte de mi paciente como un fracaso profesional

Cuando asisto a un paciente terminal he pensado en la probabilidad de mi muerte

Me conmueve enfrentar a la familia de un paciente moribundo 
(se refiere a la muerte como Pasaje, como un tránsito a otro estadio) y Salida (reflejan muerte como salida y muerte como alivio) y puntajes bajos de acuerdo en la de Aceptación (sensibilización asumir la muerte y aceptarla como algo natural y para crecimiento personal), cabe señalar que en la sub escala de Perspectivas Profesionales (actitudes ante la muerte) se contemplan porcentajes de desacuerdo y acuerdo más equilibrados. La mayoría de los sujetos, reporta que ha vivido la muerte de sus pacientes como un fracaso medad $(87,1 \%)$ y miedo a que la vida llegue a su fin $(96,8 \%)$.

Las correlaciones más significativas con respecto a las Actitudes ante la muerte que presento el personal de Cuidados Paliativos, se muestran en la tabla 4 , en la que existe una correlación moderada entre considerar morboso pensar deliberadamente en su inevitable muerte y el sexo $(0,525)$. Existe una correlación directamente proporcional moderada entre el no se puede evitar pensar en su familia ante un paciente que va a morir y el tiem-

\section{Figura 1. Porcentajes de las 3 sub-escalas de la Escala de Ansiedad ante la Muerte de Templer}



profesional, que el más alto sentido de su trabajo es salvar la vida de un paciente y que no quisieran asistir al paciente el episodio final de su vida.

Con respecto a los resultados obtenidos en la Escala de Ansiedad ante la Muerte de Templer, y de acuerdo a la interpretación de los resultados basados en Rivera-Ledesma y Montero-López ${ }^{(30)}$ en la Figura 1 se puede observar que la mayoría de los sujetos presentan baja ansiedad en las tres sub escalas: miedo a la muerte $(90,3 \%)$, miedo a la agonía o a la enfer- po dedicado a la atención del paciente con cáncer $(0,416)$ y una correlación inversamente proporcional moderada con respecto al grado de estudios $(-0,409)$ y una correlación baja con los años de experiencia clínica $(-0,379)$, con un nivel de significancia de 0,01.

En la tabla 5 que muestra la correlación entre las creencias acerca del paciente terminal y las variables generales y profesionales con un nivel de significancia del 0.01 ; se encontró que, existe una correlación directamente proporcional moderada 


\section{Tabla 4. Correlación entre la Escala de Actitudes ante la Muerte, variables generales (sexo) y variables profesiones}

Variables

Generales
Variables

Profesionales
6PP He vivido la muerte de mi paciente como un fracaso profesional

Coeficiente de Correlación

Sig. (bilateral)

0,358

Nivel se significancia
0,48

0,05

8E Considero morboso pensar deliberada-

mente en mi inevitable muerte

Coeficiente de Correlación

0,525

Sig. (bilateral)

0,002

Nivel se significancia

0,01

11PP No puedo evitar pensar en mi fami-

lia ante un paciente que va a morir

Coeficiente de Correlación

Sig. (bilateral)

Nivel se significancia

24 Encuentro difícil encarar la muerte

Coeficiente de Correlación

$\begin{array}{ccc}0,376 & -0,409 & -0,379 \\ 0,037 & 0,022 & 0,035 \\ 0,05 & 0,05 & 0,05\end{array}$

Sig. (bilateral)

Nivel se significancia

0,05

0,05

entre considerar que la morfina se utiliza para el tratamiento del dolor y el tiempo de atención al paciente terminal $(0,433)$; una correlación directamente proporcional moderada entre considerar a la boca seca, el estreñimiento, el cansancio y el desgano como síntomas habituales en el paciente terminal y edad $(0,473)$, años de experiencia $(0,515)$ y tiempo de atención al paciente terminal $(0,554)$.

Finalmente con respecto a las correlaciones entre la ansiedad ante la muerte y las variables personales y profesionales (tabla 6) se encontró que existe una co- 


\title{
Tabla 5. Correlación entre la Escala de Creencias con relación al paciente terminal y las variables generales y profesionales
}

\author{
Variables Generales \\ Variables \\ Profesionales
}

\begin{tabular}{|c|c|c|c|c|}
\hline Reactivos & Edad & $\begin{array}{c}\text { Estado } \\
\text { Civil }\end{array}$ & $\begin{array}{l}\text { Años de } \\
\text { Experiencia } \\
\text { clínica }\end{array}$ & $\begin{array}{l}\text { Tiempo de aten- } \\
\text { ción al paciente } \\
\text { terminal (meses) }\end{array}$ \\
\hline
\end{tabular}

(8) La morfina se utiliza para el tratamiento del dolor
Coeficiente de Correlación
0,433
Sig. (bilateral)
0,015
Nivel se significancia
0,05

(11) La boca seca, el estreñimiento, el cansancio y el desgano son síntomas habituales en el paciente terminal
Coeficiente de Correlación
0,473
0,357
0,515
0,554
Sig. (bilateral)
0,007
0,49
0,003
0,001
Nivel se significancia
0,01
0,05
0,01
0,01

(15) Lo que más le preocupa al enfermo terminal en el proceso del morir es el control de síntomas

Coeficiente de Correlación

0,372

Sig. (bilateral)

0,039

Nivel se significancia

0,05

\section{Tabla 6. Correlación entre la Escala de Ansiedad ante la Muerte de Templer y Variables generales (Sexo)}

\section{Variables generales}

Sexo

Coeficiente de Correlación

Sig. (bilateral)

Nivel se significancia
0,428

0,016

Miedo a la muerte

0,01 
rrelación directamente proporcional moderada entre el miedo a la muerte y el sexo $(0,428)$ con un nivel de significancia del 0,01 .

\section{DISCUSIÓN}

Dentro de las generalidades de la muestra estudiada recién conformada, en una unidad de Cuidados Paliativos, cabe señalar que se trata de una población joven que de los 20 a los 40 años, en su mayoría mujeres solteras con grado de estudios de licenciatura en ciencias de la salud (medicina y psicología); con experiencia en el campo clínico de menos de 10 años, en la atención del paciente oncológico con menos de 5 años y en la atención de pacientes terminales en menos de 12 meses. Lo cual nos permite concluir que es personal de salud muy joven quién se está integrado a los equipos de cuidados paliativos y que son predominantemente de sexo femenino que seguramente tiene que ver con el sistema de crianza en la población mexicana donde resalta el sistema matriarcado.

Con respecto a las creencias acerca del paciente terminal podemos concluir que en la población estudiada no existe una tendencia significativa con respecto a considerar que el dolor es un síntoma de difícil control, el informar o no al paciente terminal sobre su pronóstico y a que lo que más le preocupa al paciente son los aspectos emocionales. Existe una alta tendencia en considerar que tanto para el paciente como para la familia lo mejor es morir en casa, que la morfina es un tratamiento adecuado para el dolor por cáncer en fase avanzada y que el paciente debe recibir además de la atención médica, apoyo psicológico y espiritual. De igual manera existe una alta tendencia en la identificación de síntomas como boca seca, estreñimiento, cansancio y desgano como síntomas del paciente terminal, siendo además estos síntomas, la principal preocupación del paciente en el proceso del morir, sin embargo cabe señalar que dicha consideración depende de la profesión, grado de estudios y años de experiencia clínica; ya que entre menor sea el grado de estudios y los años de contacto clínico con el paciente oncológico y/o terminal menos reconocimiento y a atención se tiene a estos síntomas.

Dentro de las actitudes con respecto a la muerte podemos concluir que el personal de salud que proporciona la atención al paciente terminal considera que debemos pensar en la muerte y no evitarlo, sino considerarla como una posibilidad real y no solo por la edad o por la evolución del ser humano. Dentro de las actitudes más significativas son aquellas en las que el personal de salud considera que al estar en contacto con el paciente terminal les ha generado mayor responsabilidad y atención hacia la vida, por lo corta que puede resultar y han reconocido que la muerte ha promovido el crecimiento personal y les ha permitido aceptar su propia muerte, aun cuando existe ansiedad ante esta posibilidad, y que han Ilegado a concluir que la muerte no es una salida, aún ante lo complicado y difícil que pueda resultar la vida.

La muerte en el personal de cuidados paliativos no se vive como un fracaso profesional, sino que los ha llevado a verla como algo natural, sin embargo no siempre pueden evitar pensar en la posibilidad de su propia muerte o de algún familiar cercano; al respecto Grau, Abalo et al. ${ }^{(20)}$ también identificaron que los profesionales de cuidados paliativos ofrecen menos respuesta defensivas ante su acercamiento profesional a la atención de los enfermos pero mantienen respuestas relativamente evasivas cargadas de temor y ambigüedad y en donde predominan los componentes afectivo-emocionales con respecto a las actitudes ante la muerte. 
Es importante resaltar que no existe una tendencia definida con respecto a considerar a la muerte como tránsito, sin embargo surge un cuestionamiento en general en el personal de salud: ¿qué sucede en el momento y después de la muerte?

Lo cual nos lleva a reflexionar sobre la necesidad de indagar más acerca la espiritualidad en el personal de salud, principalmente aquellos que están cotidianamente en contacto con la muerte y desarrollar programas educativos con respecto al manejo de la muerte haciendo énfasis en la calidad humana y profesional del personal de salud como lo establece Tomás-Sábado $^{(12)}$ y proponemos también retomar la espiritualidad y el manejo de las emociones.

Dentro de las limitaciones más importantes del estudio se encuentra que fue una muestra muy reducida de participantes, sin embargo cabe señalar que fue una muestra cautiva por lo que es recomendable poder continuar realizando estudios longitudinales para tener mayor información con respecto a las actitudes, creencias y ansiedad ante la muerte del personal de salud que se encuentra en formación.

Se recomienda también continuar con el diseño o adaptación de instrumentos metodológicamente válidos para población mexicana y poder dar continuidad a este tipo de temas con el personal de salud.

\section{AGRADECIMIENTOS}

La presente investigación está registrada en los Comités de Investigación y de Bioética del Instituto Nacional de Cancerología con el núm. 011031POI / CB719.

\section{REFERENCIAS BIBLIOGRÁFICAS}

1. Martin C, Julian R. Causes of stress and burnout in physicians caring for the chronically and terminally ill. In: Paradis L, editor. Stress and burnout among providers caring for the terminally ill and their families. Nueva York: The Hawthorn Press Ins, 1987. p.121-46.

2. Tomas-Sábado J, Gómez J. Variables relacionadas con la ansiedad ante la muerte. Rev Psicol Gen Apl 2003;55:257-79.

3. Templer, D. The Construction and validation of a Death Anxiety Scale. J Gen Psychol 1970;82:165-77.

4. Malishev M. El sentido de la muerte. Ciencia Ergo Sum. 2003; 10: 51-58.

5. Aries, P. Historia de la muerte en occidente. Barcelona: El Acantilado, 2000.

6. Wong FKY, Lee WM Mok E. Educating nurses to care for the dying in Hong Kong. Cancer Nurs 2001; 24: 112-21.

7. Barreto P. Cuidados paliativos al enfermo de sida en situación terminal: enfoque multidisciplinar. Rev Psicol Gen Apl 1994; 2: 201-208

8. Widder J, Glawischning-Goschnik M. The concept of disease in palliative medicine. Med Health Care Philos 2002; 5: 191-197.

9. Vitello-Cicciu JM. Exploring emotional intelligence. Implications for nursing leaders. J Nurs Adm 2002;32:203-10

10. Centeno C. Principios de medicina paliativa. En: López F, editor. Manual de oncología clínica. Valladolid: Secretariado de publicaciones de la Universidad de Valladolid, 1999.

11. Tomás-Sábado J, Guix E. Ansiedad ante la muerte: efectos de un curso de formación en enfermeras y auxiliares de enfermería. Enferm Clin 2001;11:104-9.

12. Tomás-Sábado J. Construcción y validación de una escala de ansiedad ante la muerte [Tesis Doctoral]. Barcelona: Universidad de Barcelona, 2001.

13. Mok E, Lee WM, Wong FKY. The issue of death and dying: employing problembased learning in nursing education. Nurse Educ Today 2002;22:319-29.

14. Gala J, Lupiani M, Raja, R, Guillén C, González Infante JM, Villaverde MC, Alba I. Actitudes psicológicas ante la muerte y el duelo. Una revisión conceptual. Cuadernos de Medicina Forense 2002;30: 39-50. 
15. Pichardo G. Diner K. La experiencia de la muerte y los cuidados paliativos. Una visión desde enfermería. Anales Médicos 2010;55:161-6.

16. Brockopp DY, King DB, Hamilton JE. The dying patient: A comparative study of nurse caregiver characteristics. Death Stud 1991;15: 245-58.

17. Collel R, Limonero J, Otero M. Actitudes y emociones en estudiantes de enfermería ante la muerte y la enfermedad terminal. Investigación en salud, 2003; V: 0.

18. Gómez Sancho M. Morir con dignidad. Madrid: Arán Ediciones, 2005.

19. Fonnegra I. La muerte, el morir y el deudo desde diferentes perspectivas. Folleto de la fundación Omega, Bogotá, 1992.

20. Grau J, Llantá M, Massip C, Chacón M, Reyes C, Infante $\mathrm{O}$, et al. Ansiedad y actitudes ante la muerte: revisión y caracterización en un grupo heterogéneo de profesionales que se capacita en cuidados paliativos. Pensamiento Psicológico 2008;4:27-58.

21. Lara K, Ruiz N, Balcázar P. Ansiedad ante la muerte en adultos de población general y personal que trabaja en el sector salud. Revista Científica Electrónica de Psicología ICSa-UAEH. 2009; 8: 83-106.

22. Stevens SJ, Cooper PE, Thomas LE. Age norms for Templer's Death Anxiety Scale. Psychol Reports 1980;46:205-6.

23. Benítez M, Asensio A. Fundamentos y objetivos de los cuidados paliativos. Aten Primaria 2002;1:50-2.
24. Escot C, Artero S, Gandubert C, Boulenberg J, Ritchie K. Stress levels in nursing staff working in oncology. Stress Health 2001;5:273-9.

25. Ortega C, López F. Intervención psicológica en cuidados paliativos: revisión y perspectivas. Clin Salud 2005;16:143-60.

26. Villoro L. Creer, saber, conocer, México; México: Siglo XXI, 1982. En: Ortiz-MiIlán G. Una definición muy general. Un comentario a la definición de Creencia de Valloro. Universidad Autónoma Metropolitana: Signos Filosóficos. 2007; IX: 181-9.

27. Tomás-Sábado J, Gomez-Benito J. Psychometric properties of the Spanish form of Templer's Death Anxiety Scale. Psychol Reports 2002; 91: 1116-20.

28. Lambreth J. Psicología Social. Madrid: Pirámide, 1982.

29. Hernández G, González V, Fernández L, Infante $\mathrm{O}$. Actitud ante la muerte en los médicos de la familia. Rev Cubana Med Gen Integr 2002;18: 22-32.

30. Colell R. Análisis de las Actitudes ante la muerte y el enfermo al final de la vida en estudiantes de enfermería de Andalucía y Cataluña. Tesis Doctoral: Universidad Autónoma de Barcelona, 2005.

31. Rivera-Ledezma A, Montero- López M. Propiedades psicométricas de la escala de ansiedad ante la muerte de Templer en sujetos mexicanos. Drivers: Perspect Psicol 2011; 6:135-140. Doi: 10.5460/jbhsi. v2.2.26795. 
